\title{
ON EULER MIDPOINT FORMULAE
}

\author{
LJ. DEDIĆ ${ }^{1}$, M. MATIĆ ${ }^{1}$ and J. PEČARIĆ ${ }^{2}$
}

(Received 19 January, 2000; revised 6 November, 2004)

\begin{abstract}
Modified versions of the Euler midpoint formula are given for functions whose derivatives are either functions of bounded variation, Lipschitzian functions or functions in $L_{p}$-spaces. The results are applied to quadrature formulae.
\end{abstract}

\section{Introduction}

S. S. Dragomir, P. Cerone and A. Sofo [6] proved the inequalities

$$
\left|\int_{a}^{b} f(t) d t-(b-a) f\left(\frac{a+b}{2}\right)\right| \leq\left\{\begin{array}{l}
\frac{(b-a)^{3}}{24}\left\|f^{\prime \prime}\right\|_{\infty}, \\
\frac{(b-a)^{2+1 / q}}{8(2 q+1)^{1 / q}}\left\|f^{\prime \prime}\right\|_{p}, \\
\frac{(b-a)^{2}}{8}\left\|f^{\prime \prime}\right\|_{1},
\end{array}\right.
$$

where $f^{\prime \prime}$ belongs to the appropriate space, $1 / p+1 / q=1$ and $p>1$. The first of these is usually referred to as the midpoint inequality. A simple application of the midpoint inequality is as follows. Divide the interval $[a, b]$ into $v$ subintervals of equal length $h=(b-a) / \nu$. For given $f:[a, b] \rightarrow \mathbb{B}$, consider the midpoint quadrature formula

$$
\int_{a}^{b} f(t) d t=S_{v}(f)+\rho_{\nu}(f)
$$

\footnotetext{
'Department of Mathematics, Faculty of Natural Sciences, Mathematics and Education, University of Split, Teslina 12,21000 Split, Croatia; e-mail: ljuban@pmfst.hr and mmatic@pmfst.hr.

${ }^{2}$ Faculty of Textile Technology, University of Zagreb, Pierottijeva 6, 10000 Zagreb, Croatia; e-mail: pecaric@element.hr.

(C) Australian Mathematical Society 2005, Serial-fee code 1446-1811/05
} 
where

$$
S_{\nu}(f):=h \sum_{i=1}^{\nu} f\left(a+(2 i-1) \frac{h}{2}\right) .
$$

If $f$ is twice differentiable and $\left\|f^{\prime \prime}\right\|_{\infty}<\infty$, then applying the midpoint inequality gives the estimate $\left|\rho_{\nu}(f)\right| \leq\left(\nu h^{3} / 24\right)\left\|f^{\prime \prime}\right\|_{\infty}$ for the remainder $\rho_{\nu}(f)$.

A generalisation of (1.1) for $n$-times differentiable functions has been obtained in the recent paper [6]. In this paper we give another generalisation of (1.1) and use it to establish various error estimates for some quadrature rules which generalise the midpoint quadrature rule (1.2).

In the recent paper [4] we proved the formula

$$
\begin{aligned}
f(x)= & \frac{1}{b-a} \int_{a}^{b} f(t) d t+\sum_{k=1}^{n-1} \frac{(b-a)^{k-1}}{k !} B_{k}\left(\frac{x-a}{b-a}\right)\left[f^{(k-1)}(b)-f^{(k-1)}(a)\right] \\
& -\frac{(b-a)^{n-1}}{n !} \int_{[a, b]}\left[B_{n}^{*}\left(\frac{x-t}{b-a}\right)-B_{n}\left(\frac{x-a}{b-a}\right)\right] d f^{(n-1)}(t) .
\end{aligned}
$$

Here $B_{k}(t)$ are the Bernoulli polynomials, $B_{k}=B_{k}(0)$ the Bernoulli numbers, and $B_{k}^{*}(t), k \geq 0$, are periodic functions of period one, related to the Bernoulli polynomials by

$$
\begin{aligned}
B_{k}^{*}(t)=B_{k}(t), & \text { for } 0 \leq t<1, \\
B_{k}^{*}(t+1)=B_{k}^{*}(t), & \text { for } t \in \mathbb{R},
\end{aligned}
$$

so that $B_{0}^{*}(t)=1, B_{1}^{*}(t)$ is a discontinuous function with a jump of -1 at each integer and $B_{k}^{*}(t), k \geq 2$, is a continuous function. The sum in (1.3) is taken as zero when $n=1$. The Bernoulli polynomials $B_{k}(t), k \geq 0$, are uniquely determined by the identities

$$
B_{k}^{\prime}(t)=k B_{k-1}(t), \quad k \geq 1, \quad B_{0}(t)=1
$$

and

$$
B_{k}(t+1)-B_{k}(t)=k t^{k-1}, k \geq 0
$$

For further details on the Bernoulli polynomials and the Bernoulli numbers see [1] or [2]. The formula (1.3) is valid for every function $\mathrm{r} f:[a, b] \rightarrow \mathbb{R}$ such that $f^{(n-1)}$ is a continuous function of bounded variation on $[a, b]$ for some $n \geq 1$, and for every $x \in[a, b]$. Also this formula is an extension of a Krylov formula [7, page 17].

In Section 2 we make use of (1.3) to give modified versions of the Euler midpoint formula. In Section 3 we use these modified Euler midpoint formulae to prove some generalisations of (1.1) for functions whose derivatives are either functions of 
bounded variation, Lipschitzian functions or functions from $L_{p}$-spaces. Finally, in Section 4, we consider the repeated Euler midpoint formula and the repeated modified Euler formula which generalise the midpoint quadrature formula (1.2). Applying the estimates obtained in Section 3, we establish various error estimates for these generalised quadrature formulae.

\section{The Euler midpoint formulae}

THEOREM 2.1. Let $f:[a, b] \rightarrow \mathbb{R}$ be such that $f^{(n-1)}$ is a continuous function of bounded variation on $[a, b]$ for some $n \geq 1$. For any integer $m$ such that $0 \leq m \leq n / 2$ define

$$
T_{m}(f):=\sum_{k=1}^{m} \frac{(b-a)^{2 k}}{(2 k) !} B_{2 k}\left[f^{(2 k-1)}(b)-f^{(2 k-1)}(a)\right],
$$

with the convention that the sum is zero when $m=0$, that is, $T_{0}(f)=0$. If $n=2 r-1$, $r \geq 1$, then

$$
\begin{aligned}
\int_{a}^{b} f(t) d t= & (b-a) f\left(\frac{a+b}{2}\right)-T_{r-1}(f) \\
& +\frac{(b-a)^{2 r-1}}{(2 r-1) !} \int_{[a, b]} B_{2 r-1}^{*}\left(\frac{(a+b) / 2-t}{b-a}\right) d f^{(2 r-2)}(t) .
\end{aligned}
$$

If $n=2 r, r \geq 1$, then

$$
\begin{aligned}
\int_{a}^{b} f(t) d t= & (b-a) f\left(\frac{a+b}{2}\right)-T_{r-1}(f) \\
& +\frac{(b-a)^{2 r}}{(2 r) !} \int_{[a, b]}\left[B_{2 r}^{*}\left(\frac{(a+b) / 2-t}{b-a}\right)-B_{2 r}\left(\frac{1}{2}\right)\right] d f^{(2 r-1)}(t)
\end{aligned}
$$

and

$$
\begin{aligned}
\int_{a}^{b} f(t) d t= & (b-a) f\left(\frac{a+b}{2}\right)-T_{r}(f) \\
& +\frac{(b-a)^{2 r}}{(2 r) !} \int_{|a, b|} B_{2 r}^{*}\left(\frac{(a+b) / 2-t}{b-a}\right) d f^{(2 r-1)}(t) .
\end{aligned}
$$

PROOF. Set $x=(a+b) / 2$ in (1.3) and multiply by $b-a$ to obtain the identity

$$
\begin{aligned}
(b-a) f\left(\frac{a+b}{2}\right)= & \int_{a}^{b} f(t) d t+\sum_{k=1}^{n-1} \frac{(b-a)^{k}}{k !} B_{k}\left(\frac{1}{2}\right)\left[f^{(k-1)}(b)-f^{(k-1)}(a)\right] \\
& -\frac{(b-a)^{n}}{n !} \int_{[a, b]}\left[B_{n}^{*}\left(\frac{(a+b) / 2-t}{b-a}\right)-B_{n}\left(\frac{1}{2}\right)\right] d f^{(n-1)}(t) .
\end{aligned}
$$


We have $[1,23.1 .19,23.1 .21] B_{2 k-1}(1 / 2)=0, k \geq 1$, so that the above identity can be rewritten as

$$
\begin{aligned}
\int_{a}^{b} f(t) d t= & (b-a) f\left(\frac{a+b}{2}\right) \\
& -\sum_{k=1}^{[(n-1) / 2]} \frac{(b-a)^{2 k}}{(2 k) !} B_{2 k}\left(\frac{1}{2}\right)\left[f^{(2 k-1)}(b)-f^{(2 k-1)}(a)\right] \\
& +\frac{(b-a)^{n}}{n !} \int_{[a, b]}\left[B_{n}^{*}\left(\frac{(a+b) / 2-t}{b-a}\right)-B_{n}\left(\frac{1}{2}\right)\right] d f^{(n-1)}(t),
\end{aligned}
$$

where $[n]$ denotes the greatest integer less than or equal to $n$. The sum on the righthand side of (2.5) is taken to be zero for $n=1$ or $n=2$. If $n=2 r-1, r \geq 1$, then $[(n-1) / 2]=r-1, B_{2 r-1}(1 / 2)=0$ and (2.5) becomes (2.2). If $n=2 r, r \geq 1$, then again $[(n-1) / 2]=r-1$ and (2.5) reduces to (2.3). Finally, note that

$$
\begin{aligned}
\int_{[a, b]} & {\left[B_{2 r}\left(\frac{(a+b) / 2-t}{b-a}\right)-B_{2 r}\left(\frac{1}{2}\right)\right] d f^{(2 r-1)}(t) } \\
= & \int_{[a, b]} B_{2 r}\left(\frac{(a+b) / 2-t}{b-a}\right) d f^{(2 r-1)}(t)-B_{2 r}\left(\frac{1}{2}\right)\left[f^{(2 r-1)}(b)-f^{(2 r-1)}(a)\right]
\end{aligned}
$$

and

$$
-T_{r-1}(f)-\frac{(b-a)^{2 r}}{(2 r) !} B_{2 r}\left(\frac{1}{2}\right)\left[f^{(2 r-1)}(b)-f^{(2 r-1)}(a)\right]=-T_{r}(f),
$$

so that (2.3) can be rewritten as (2.4).

REMARK 1 . In the case when $F:[a, b] \rightarrow \mathbb{R}$ is such that $F^{\prime}$ exists and is integrable on $[a, b]$, then the Riemann-Stieltjes integral $\int_{[a, b]} G(t) d F(t)$ is equal to the Riemann integral $\int_{a}^{b} G(t) F^{\prime}(t) d t$. Therefore, if $f^{(2 r-1)}$ exists for some $r \geq 1$ and is integrable on $[a, b]$, then $(2.2)$ reduces to

$$
\begin{aligned}
\int_{a}^{b} f(t) d t= & (b-a) f\left(\frac{a+b}{2}\right)-T_{r-1}(f) \\
& +\frac{(b-a)^{2 r-1}}{(2 r-1) !} \int_{[a, b]} B_{2 r-1}^{*}\left(\frac{(a+b) / 2-t}{b-a}\right) f^{(2 r-1)}(t) d t
\end{aligned}
$$

Similarly, if $f^{(2 r)}$ exists for some $r \geq 1$ and is integrable on $[a, b]$, then (2.3) and (2.4) reduce to

$$
\begin{aligned}
\int_{a}^{b} f(t) d t= & (b-a) f\left(\frac{a+b}{2}\right)-T_{r-1}(f) \\
& +\frac{(b-a)^{2 r}}{(2 r) !} \int_{[a . b]}\left[B_{2 r}^{*}\left(\frac{(a+b) / 2-t}{b-a}\right)-B_{2 r}\left(\frac{1}{2}\right)\right] f^{(2 r)}(t) d t
\end{aligned}
$$


and

$$
\begin{aligned}
\int_{a}^{b} f(t) d t= & (b-a) f\left(\frac{a+b}{2}\right)-T_{r}(f) \\
& +\frac{(b-a)^{2 r}}{(2 r) !} \int_{[a, b]} B_{2 r}^{*}\left(\frac{(a+b) / 2-t}{b-a}\right) f^{(2 r)}(t) d t
\end{aligned}
$$

respectively.

\section{Generalisations of the midpoint inequality}

THEOREM 3.1. Let $f:[a, b] \rightarrow \mathbb{R}$ be such that $f^{(n-1)}$ is an L-Lipschitzian function on $[a, b]$ for some $n \geq 1$. Assume the notation established by (2.1). If $n=2 r-1$, $r \geq 1$, then

$$
\left|\int_{a}^{b} f(t) d t-(b-a) f\left(\frac{a+b}{2}\right)+T_{r-1}(f)\right| \leq \frac{(b-a)^{2 r}}{(2 r) !} 4\left(1-2^{-2 r}\right)\left|B_{2 r}\right| L .
$$

If $n=2 r, r \geq 1$, then

$$
\left|\int_{a}^{b} f(t) d t-(b-a) f\left(\frac{a+b}{2}\right)+T_{r-1}(f)\right| \leq \frac{(b-a)^{2 r+1}}{(2 r) !}\left(1-2^{1-2 r}\right)\left|B_{2 r}\right| L .
$$

Also, we have

$$
\begin{aligned}
& \left|\int_{a}^{b} f(t) d t-(b-a) f\left(\frac{a+b}{2}\right)+T_{r}(f)\right| \\
& \quad \leq \frac{(b-a)^{2 r+1}}{(2 r) !} \int_{0}^{1}\left|B_{2 r}(t)\right| d t L \leq \frac{(b-a)^{2 r+1}}{(2 r) !} 2\left|B_{2 r}\right| L .
\end{aligned}
$$

PROOF. For an integrable function $F:[a, b] \rightarrow \mathbb{R}$ we have

$$
\left|\int_{[a, b]} F(t) d f^{(n-1)}(t)\right| \leq \int_{a}^{b}|F(t)| d t L
$$

since $f^{(n-1)}$ is an $L$-Lipschitzian function. Applying the above estimate, we get

$$
\begin{aligned}
& \left|\frac{(b-a)^{2 r-1}}{(2 r-1) !} \int_{[a, b]} B_{2 r-1}^{*}\left(\frac{(a+b) / 2-t}{b-a}\right) d f^{(2 r-2)}(t)\right| \\
& \quad \leq \frac{(b-a)^{2 r-1}}{(2 r-1) !} \int_{a}^{b}\left|B_{2 r-1}^{*}\left(\frac{(a+b) / 2-t}{b-a}\right)\right| d t L \\
& \quad=\frac{(b-a)^{2 r}}{(2 r-1) !} \int_{-1 / 2}^{1 / 2}\left|B_{2 r-1}^{*}(t)\right| d t L=\frac{(b-a)^{2 r}}{(2 r-1) !} \int_{0}^{1}\left|B_{2 r-1}(t)\right| d t L .
\end{aligned}
$$


Because of

and

$$
0<(-1)^{r} B_{2 r-1}(t)<\frac{2(2 r-1) !}{(2 \pi)^{2 r-1}\left(1-2^{2-2 r}\right)}, \quad 0<t<1 / 2, r \geq 2
$$

$$
B_{2 r-1}(1-t)=-B_{2 r-1}(t), \quad t \in[0,1]
$$

(see $[1,23.1 .14,23.1 .8])$, we have

$$
\begin{aligned}
\int_{0}^{1}\left|B_{2 r-1}(t)\right| d t & =2 \int_{0}^{1 / 2}\left|B_{2 r-1}(t)\right| d t \\
& =2\left|\int_{0}^{1 / 2} B_{2 r-1}(t) d t\right|=\frac{1}{r}\left|B_{2 r}(1 / 2)-B_{2 r}(0)\right| \\
& =\frac{1}{r}\left|-\left(1-2^{1-2 r}\right) B_{2 r}-B_{2 r}\right|=\frac{\left(2-2^{1-2 r}\right)}{r}\left|B_{2 r}\right| .
\end{aligned}
$$

In the above calculation we used the identity (1.4) and

$$
B_{2 r}(1 / 2)=-\left(1-2^{1-2 r}\right) B_{2 r}
$$

(see $[1,23.1 .21])$. The above estimate, in combination with (2.2), proves the inequality (3.1). Further, using the estimate (3.4), we get

$$
\begin{gathered}
\left|\frac{(b-a)^{2 r}}{(2 r) !} \int_{[a, b]}\left[B_{2 r}^{*}\left(\frac{(a+b) / 2-t}{b-a}\right)-B_{2 r}\left(\frac{1}{2}\right)\right] d f^{(2 r-1)}(t)\right| \\
\quad \leq \frac{(b-a)^{2 r}}{(2 r) !} \int_{a}^{b}\left|B_{2 r}^{*}\left(\frac{(a+b) / 2-t}{b-a}\right)-B_{2 r}\left(\frac{1}{2}\right)\right| d t L \\
\quad=\frac{(b-a)^{2 r+1}}{(2 r) !} \int_{-1 / 2}^{1 / 2}\left|B_{2 r}^{*}(t)-B_{2 r}\left(\frac{1}{2}\right)\right| d t L \\
\quad=\frac{(b-a)^{2 r+1}}{(2 r) !} \int_{0}^{1}\left|B_{2 r}(t)-B_{2 r}\left(\frac{1}{2}\right)\right| d t L .
\end{gathered}
$$

Because of

$$
(-1)^{r-1}\left(B_{2 r}(t)-B_{2 r}(1 / 2)\right) \geq 0, \quad 0 \leq t \leq 1
$$

(see [7, Section 1.2]), on using (3.7) we get

$$
\begin{aligned}
\int_{0}^{1}\left|B_{2 r}(t)-B_{2 r}(1 / 2)\right| d t & =\left|\int_{0}^{1}\left(B_{2 r}(t)-B_{2 r}(1 / 2)\right) d t\right| \\
& =\left|B_{2 r}(1 / 2)\right|=\left(1-2^{1-2 r}\right)\left|B_{2 r}\right| .
\end{aligned}
$$


This, in combination with (2.3), proves the inequality (3.2). Finally, we can apply the estimate (3.4) to obtain

$$
\begin{aligned}
& \left|\frac{(b-a)^{2 r}}{(2 r) !} \int_{[a, b]} B_{2 r}^{*}\left(\frac{(a+b) / 2-t}{b-a}\right) d f^{(2 r-1)}(t)\right| \\
& \quad \leq \frac{(b-a)^{2 r}}{(2 r) !} \int_{a}^{b}\left|B_{2 r}^{*}\left(\frac{(a+b) / 2-t}{b-a}\right)\right| d t L \\
& \quad=\frac{(b-a)^{2 r+1}}{(2 r) !} \int_{-1 / 2}^{1 / 2}\left|B_{2 r}^{*}(t)\right| d t L=\frac{(b-a)^{2 r+1}}{(2 r) !} \int_{0}^{1}\left|B_{2 r}(t)\right| d t L
\end{aligned}
$$

which is, by (2.4), the first inequality in (3.3). Further, because of

$$
(-1)^{r}\left(B_{2 r}(t)-B_{2 r}\right)>0, \quad 0<t<1
$$

(see [7, Section 1.2]), we have

and

$$
\int_{0}^{1}\left|B_{2 r}(t)-B_{2 r}\right| d t=\left|\int_{0}^{1}\left(B_{2 r}(t)-B_{2 r}\right) d t\right|=\left|B_{2 r}\right|
$$

$$
\begin{aligned}
\int_{0}^{1}\left|B_{2 r}(t)\right| d t & =\int_{0}^{1}\left|B_{2 r}(t)-B_{2 r}+B_{2 r}\right| d t \\
& \leq \int_{0}^{1}\left|B_{2 r}(t)-B_{2 r}\right| d t+\left|B_{2 r}\right|=2\left|B_{2 r}\right|
\end{aligned}
$$

which proves the second inequality in (3.3).

As corollaries to the preceding theorem let us state some particular results. Note that $B_{2}(1 / 2)=-1 / 12, B_{4}(1 / 2)=7 / 240$ and

$$
T_{0}(f)=0, \quad T_{1}(f)=-\frac{(b-a)^{2}}{24}\left[f^{\prime}(b)-f^{\prime}(a)\right] .
$$

COROLlaRY 3.2. Let $f:[a, b] \rightarrow \mathbb{B}$ be a given function. If $f$ is L-Lipschitzian on $[a, b]$, then

$$
\left|\int_{a}^{b} f(t) d t-(b-a) f\left(\frac{a+b}{2}\right)\right| \leq \frac{(b-a)^{2}}{4} L .
$$

If $f^{\prime}$ is L-Lipschitzian on $[a, b]$, then

$$
\left|\int_{a}^{b} f(t) d t-(b-a) f\left(\frac{a+b}{2}\right)\right| \leq \frac{(b-a)^{3}}{24} L .
$$


PROOF. Set $r=1$ in (3.1) to obtain the first inequality. The second one follows from (3.2) with $r=1$.

REMARK 2. The first inequality established in the above corollary has been proved by Dragomir in [5] (see also the recent survey paper [3]), while the second extends the first inequality in (1.1) to a wider class of functions. Namely, if $f$ is such that $f^{\prime \prime}$ exists and is bounded, then the second inequality from Corollary 3.2 applies with $L=\left\|f^{\prime \prime}\right\|_{\infty}$.

COROLLARY 3.3. Let $f:[a, b] \rightarrow \mathbb{R}$ be a given function. If $f^{\prime}$ is L-Lipschitzian on $[a, b]$, then

$$
\left|\int_{a}^{b} f(t) d t-(b-a) f\left(\frac{a+b}{2}\right)-\frac{(b-a)^{2}}{24}\left[f^{\prime}(b)-f^{\prime}(a)\right]\right| \leq \frac{(b-a)^{3}}{18 \sqrt{3}} L .
$$

If $f^{\prime \prime}$ is L-Lipschitzian on $[a, b]$, then

$$
\left|\int_{a}^{b} f(t) d t-(b-a) f\left(\frac{a+b}{2}\right)-\frac{(b-a)^{2}}{24}\left[f^{\prime}(b)-f^{\prime}(a)\right]\right| \leq \frac{(b-a)^{4}}{192} L .
$$

If $f^{\prime \prime \prime}$ is L-Lipschitzian on $[a, b]$, then

$$
\left|\int_{a}^{b} f(t) d t-(b-a) f\left(\frac{a+b}{2}\right)-\frac{(b-a)^{2}}{24}\left[f^{\prime}(b)-f^{\prime}(a)\right]\right| \leq \frac{7(b-a)^{5}}{5760} L .
$$

Proof. We have $B_{2}(t)=t^{2}-t+1 / 6$ and by a simple calculation we get $\int_{0}^{1}\left|B_{2}(t)\right| d t=1 / 9 \sqrt{3}$. To prove the first assertion we apply the first inequality in (3.3) with $r=1$. The second inequality follows from (3.1) with $r=2$, while the third follows from (3.2) with $r=2$.

THEOREM 3.4. Let $f:[a, b] \rightarrow \mathbb{R}$ be such that $f^{(n-1)}$ is a continuous function of bounded variation on $[a, b]$ for some $n \geq 1$. Assume the notation established by (2.1) and denote by $V_{a}^{b}\left(f^{(n-1)}\right)$ the total variation of $f^{(n-1)}$ on $[a, b]$. If $n=2 r-1, r \geq 2$, then

$$
\begin{aligned}
& \left|\int_{a}^{b} f(t) d t-(b-a) f\left(\frac{a+b}{2}\right)+T_{r-1}(f)\right| \\
& \quad \leq \frac{(b-a)^{2 r-1}}{(2 r-1) !} \max _{t \in[0.1]}\left|B_{2 r-1}(t)\right| V_{a}^{b}\left(f^{(2 r-2)}\right) \\
& \quad \leq \frac{2(b-a)^{2 r-1}}{(2 \pi)^{2 r-1}\left(1-2^{2-2 r}\right)} V_{a}^{b}\left(f^{(2 r-2)}\right) .
\end{aligned}
$$

The first inequality holds for $r=1$ too. 
If $n=2 r, r \geq 1$, then

$$
\begin{gathered}
\left|\int_{a}^{b} f(t) d t-(b-a) f\left(\frac{a+b}{2}\right)+T_{r-1}(f)\right| \\
\quad \leq \frac{(b-a)^{2 r}}{(2 r) !} 2\left(1-2^{-2 r}\right)\left|B_{2 r}\right| V_{a}^{b}\left(f^{(2 r-1)}\right)
\end{gathered}
$$

Also, we have

$$
\left|\int_{a}^{b} f(t) d t-(b-a) f\left(\frac{a+b}{2}\right)+T_{r}(f)\right| \leq \frac{(b-a)^{2 r}}{(2 r) !}\left|B_{2 r}\right| V_{a}^{b}\left(f^{(2 r-1)}\right) .
$$

PROOF. If $F:[a, b] \rightarrow \mathbb{R}$ is bounded on $[a, b]$ and the Stieltjes integral

exists, then

$$
\int_{[a, b]} F(t) d f^{(n-1)}(t)
$$

$$
\left|\int_{[a, b]} F(t) d f^{(n-1)}(t)\right| \leq \max _{t \in[a, b]}|F(t)| V_{a}^{b}\left(f^{(n-1)}\right) .
$$

Using the above estimate we get for $r \geq 1$

$$
\begin{aligned}
& \left|\frac{(b-a)^{2 r-1}}{(2 r-1) !} \int_{[a, b]} B_{2 r-1}^{*}\left(\frac{(a+b) / 2-t}{b-a}\right) d f^{(2 r-2)}(t)\right| \\
& \leq \frac{(b-a)^{2 r-1}}{(2 r-1) !} \max _{t \in[a, b]}\left|B_{2 r-1}^{*}\left(\frac{(a+b) / 2-t}{b-a}\right)\right| V_{a}^{b}\left(f^{(2 r-2)}\right) \\
& \quad=\frac{(b-a)^{2 r-1}}{(2 r-1) !} \max _{t \in[a, b]}\left|B_{2 r-1}(t)\right| V_{a}^{b}\left(f^{(2 r-2)}\right) .
\end{aligned}
$$

By (2.2), this implies the first inequality in (3.9). Also, for $r \geq 2$, using (3.5) and (3.6) we get

$$
\max _{t \in[a, b \mid}\left|B_{2 r-1}(t)\right| \leq \frac{2(2 r-1) !}{(2 \pi)^{2 r-1}\left(1-2^{2-2 r}\right)},
$$

which implies the second inequality in (3.9). Further, using (3.12) we get

$$
\begin{gathered}
\left|\frac{(b-a)^{2 r}}{(2 r) !} \int_{[a, b]}\left[B_{2 r}^{*}\left(\frac{(a+b) / 2-t}{b-a}\right)-B_{2 r}\left(\frac{1}{2}\right)\right] d f^{(2 r-1)}(t)\right| \\
\leq \frac{(b-a)^{2 r}}{(2 r) !} \max _{t \in \mid a, b]}\left|B_{2 r}^{*}\left(\frac{(a+b) / 2-t}{b-a}\right)-B_{2 r}\left(\frac{1}{2}\right)\right| V_{a}^{b}\left(f^{(2 r-1)}\right) \\
\quad=\frac{(b-a)^{2 r}}{(2 r) !} \max _{t \in\{a, b \mid}\left|B_{2 r}(t)-B_{2 r}\left(\frac{1}{2}\right)\right| V_{a}^{b}\left(f^{(2 r-1)}\right) .
\end{gathered}
$$


Now, the fact that

$$
\max _{t \in[a, b]}\left|B_{2 r}(t)-B_{2 r}(1 / 2)\right|=\left|B_{2 r}-B_{2 r}(1 / 2)\right|=2\left(1-2^{-2 r}\right)\left|B_{2 r}\right|
$$

(see [7, Section 1.2]) together with (2.3) imply (3.10). Finally, applying the estimate (3.12), we get

$$
\begin{aligned}
& \left|\frac{(b-a)^{2 r}}{(2 r) !} \int_{[a, b]} B_{2 r}^{*}\left(\frac{(a+b) / 2-t}{b-a}\right) d f^{(2 r-1)}(t)\right| \\
& \leq \frac{(b-a)^{2 r}}{(2 r) !} \max _{t \in[a, b]}\left|B_{2 r}^{*}\left(\frac{(a+b) / 2-t}{b-a}\right)\right| V_{a}^{b}\left(f^{(2 r-1)}\right) \\
& \quad=\frac{(b-a)^{2 r}}{(2 r) !} \max _{t \in[0,1]}\left|B_{2 r}(t)\right| V_{a}^{b}\left(f^{(2 r-1)}\right) .
\end{aligned}
$$

Using $\max _{t \in[0,1]}\left|B_{2 r}(t)\right|=\left|B_{2 r}\right|$ and (2.4) gives (3.11).

COROLlARY 3.5. Let $f:[a, b] \rightarrow \mathbb{R}$ be a given function. If $f$ has bounded variation on $[a, b]$, then

$$
\left|\int_{a}^{b} f(t) d t-(b-a) f\left(\frac{a+b}{2}\right)\right| \leq \frac{b-a}{2} V_{a}^{b}(f)
$$

If $f^{\prime}$ has bounded variation on $[a, b]$, then

$$
\left|\int_{a}^{b} f(t) d t-(b-a) f\left(\frac{a+b}{2}\right)\right| \leq \frac{(b-a)^{2}}{8} V_{a}^{b}\left(f^{\prime}\right) .
$$

ProOF. To prove the first assertion, apply the first inequality in (3.9) with $r=1$ and note that $\max _{t \in[0,1]}\left|B_{1}(t)\right|=\max _{t \in[0,1]}|t-1 / 2|=1 / 2$. The second assertion follows from (3.10) with $r=1$.

REMARK 3. The first inequality of Corollary 3.5 can be found in [3], while the second extends the third inequality in (1.1) to a wider class of functions. Namely, if $f$ is such that $f^{\prime \prime}$ exists and belongs to the space $L_{1}[a, b]$, then the second inequality from Corollary 3.5 applies with $V_{a}^{b}\left(f^{\prime}\right)$ replaced by $\left\|f^{\prime \prime}\right\|_{1}$.

COROllaRY 3.6. Let $f:[a, b] \rightarrow \mathbb{B}$ be a given function. If $f^{\prime}$ has bounded variation on $[a, b]$, then

$$
\left|\int_{a}^{b} f(t) d t-(b-a) f\left(\frac{a+b}{2}\right)-\frac{(b-a)^{2}}{24}\left[f^{\prime}(b)-f^{\prime}(a)\right]\right| \leq \frac{(b-a)^{2}}{12} V_{a}^{b}\left(f^{\prime}\right) .
$$


If $f^{\prime \prime}$ has bounded variation on $[a, b]$, then

$$
\left|\int_{a}^{b} f(t) d t-(b-a) f\left(\frac{a+b}{2}\right)-\frac{(b-a)^{2}}{24}\left[f^{\prime}(b)-f^{\prime}(a)\right]\right| \leq \frac{(b-a)^{3}}{72 \sqrt{3}} V_{a}^{b}\left(f^{\prime \prime}\right) .
$$

If $f^{\prime \prime \prime}$ has bounded variation on $[a, b]$, then

$$
\left|\int_{a}^{b} f(t) d t-(b-a) f\left(\frac{a+b}{2}\right)-\frac{(b-a)^{2}}{24}\left[f^{\prime}(b)-f^{\prime}(a)\right]\right| \leq \frac{(b-a)^{5}}{384} V_{a}^{b}\left(f^{\prime \prime \prime}\right) .
$$

PROOF. The first inequality follows from (3.11) with $r=1$. To prove the second, note that $\max _{t \in[0,1]}\left|B_{3}(t)\right|=\max _{t \in[0,1]}\left|t^{3}-3 t^{2} / 2+t / 2\right|=1 / 12 \sqrt{3}$ and apply the first inequality in (3.9) with $r=2$. Finally, the third inequality follows from (3.10) with $r=2$.

THEOREM 3.7. Let $f:[a, b] \rightarrow \mathbb{R}$ be such that $f^{(n)} \in L_{1}[a, b]$ for some $n \geq 1$. Assume the notation established by (2.1). If $n=2 r-1, r \geq 2$, then

$$
\begin{aligned}
& \left|\int_{a}^{b} f(t) d t-(b-a) f\left(\frac{a+b}{2}\right)+T_{r-1}(f)\right| \\
& \quad \leq \frac{(b-a)^{2 r-1}}{(2 r-1) !} \max _{t \in[0,1]} \mid B_{2 r-1}(t)\left\|f^{(2 r-1)}\right\|_{1} \leq \frac{2(b-a)^{2 r-1}}{(2 \pi)^{2 r-1}\left(1-2^{2-2 r}\right)}\left\|f^{(2 r-1)}\right\|_{1} .
\end{aligned}
$$

The first inequality holds for $r=1$ too. If $n=2 r, r \geq 1$, then

$$
\left|\int_{a}^{b} f(t) d t-(b-a) f\left(\frac{a+b}{2}\right)+T_{r-1}(f)\right| \leq \frac{(b-a)^{2 r}}{(2 r) !} 2\left(1-2^{-2 r}\right)\left|B_{2 r}\right|\left\|f^{(2 r)}\right\|_{1} .
$$

Also, we have

$$
\left|\int_{a}^{b} f(t) d t-(b-a) f\left(\frac{a+b}{2}\right)+T_{r}(f)\right| \leq \frac{(b-a)^{2 r}}{(2 r) !}\left|B_{2 r}\right|\left\|f^{(2 r)}\right\|_{1} .
$$

PROOF. Since $f^{(n)} \in L_{1}[a, b], f^{(n-1)}$ has bounded variation on $[a, b]$ and

$$
V_{a}^{b}\left(f^{(n-1)}\right)=\int_{a}^{b}\left|f^{(n)}(t)\right| d t=\left\|f^{(n)}\right\|_{1} .
$$

Now apply Theorem 3.4 to obtain the inequalities stated in the theorem.

COROLlary 3.8. Let $f:[a, b] \rightarrow \mathbb{R}$ be a given function. If $f^{\prime} \in L_{1}[a, b]$, then

$$
\left|\int_{a}^{b} f(t) d t-(b-a) f\left(\frac{a+b}{2}\right)\right| \leq \frac{b-a}{2}\left\|f^{\prime}\right\|_{1} .
$$

If $f^{\prime \prime} \in L_{1}[a, b]$, then

$$
\left|\int_{a}^{b} f(t) d t-(b-a) f\left(\frac{a+b}{2}\right)\right| \leq \frac{(b-a)^{2}}{8}\left\|f^{\prime \prime}\right\|_{1} .
$$


PROOF. Apply Corollary 3.5 with the same argument as in the proof of the preceding theorem.

REMARK 4. The first inequality of the above corollary can be found in [3], while the second coincides with the third inequality in (1.1).

COROLlaRY 3.9. Let $f:[a, b] \rightarrow \mathbb{R}$ be a given function. If $f^{\prime \prime} \in L_{1}[a, b]$, then

$$
\left|\int_{a}^{b} f(t) d t-(b-a) f\left(\frac{a+b}{2}\right)-\frac{(b-a)^{2}}{24}\left[f^{\prime}(b)-f^{\prime}(a)\right]\right| \leq \frac{(b-a)^{2}}{12}\left\|f^{\prime \prime}\right\|_{1} \text {. }
$$

If $f^{\prime \prime \prime} \in L_{1}[a, b]$, then

$$
\left|\int_{a}^{b} f(t) d t-(b-a) f\left(\frac{a+b}{2}\right)-\frac{(b-a)^{2}}{24}\left[f^{\prime}(b)-f^{\prime}(a)\right]\right| \leq \frac{(b-a)^{3}}{72 \sqrt{3}}\left\|f^{\prime \prime \prime}\right\|_{1} .
$$

If $f^{\prime \prime \prime \prime} \in L_{1}[a, b]$, then

$$
\left|\int_{a}^{b} f(t) d t-(b-a) f\left(\frac{a+b}{2}\right)-\frac{(b-a)^{2}}{24}\left[f^{\prime}(b)-f^{\prime}(a)\right]\right| \leq \frac{(b-a)^{5}}{384}\left\|f^{\prime \prime \prime \prime}\right\|_{1} .
$$

ProOF. Apply Corollary 3.6 with the same argument as in the proof of the preceding theorem.

THEOREM 3.10. Let $(p, q)$ be a pair of conjugate exponents, that is, $1<p, q<\infty$, $1 / p+1 / q=1$ or $p=\infty, q=1$. Let $f:[a, b] \rightarrow \mathbb{R}$ be such that $f^{(n)} \in L_{p}[a, b]$ for some $n \geq 1$ and assume the notation established by (2.1). If $n=2 r-1, r \geq 1$, then

$$
\begin{aligned}
& \left|\int_{a}^{b} f(t) d t-(b-a) f\left(\frac{a+b}{2}\right)+T_{r-1}(f)\right| \\
& \quad \leq \frac{(b-a)^{2 r-1+1 / q}}{(2 r-1) !}\left(\int_{0}^{1}\left|B_{2 r-1}(t)\right|^{q} d t\right)^{1 / q}\left\|f^{(2 r-1)}\right\|_{p} .
\end{aligned}
$$

If $n=2 r, r \geq 1$, then

$$
\begin{aligned}
& \left|\int_{a}^{b} f(t) d t-(b-a) f\left(\frac{a+b}{2}\right)+T_{r-1}(f)\right| \\
& \quad \leq \frac{(b-a)^{2 r+1 / q}}{(2 r) !}\left(\int_{0}^{1}\left|B_{2 r}(t)-B_{2 r}\left(\frac{1}{2}\right)\right|^{q} d t\right)^{1 / q}\left\|f^{(2 r)}\right\|_{\rho} .
\end{aligned}
$$

Also, we have

$$
\begin{aligned}
& \left|\int_{a}^{b} f(t) d t-(b-a) f\left(\frac{a+b}{2}\right)+T_{r}(f)\right| \\
& \quad \leq \frac{(b-a)^{2 r+1 / q}}{(2 r) !}\left(\int_{0}^{l}\left|B_{2 r}(t)\right|^{q} d t\right)^{1 / q}\left\|f^{(2 r)}\right\|_{p} .
\end{aligned}
$$


[13]

On Euler midpoint formulae

429

ProOF. By applying the Hölder inequality we have

$$
\begin{aligned}
& \left|\frac{(b-a)^{2 r-1}}{(2 r-1) !} \int_{a}^{b} B_{2 r-1}^{*}\left(\frac{(a+b) / 2-t}{b-a}\right) f^{(2 r-1)}(t) d t\right| \\
& \quad \leq \frac{(b-a)^{2 r-1}}{(2 r-1) !}\left(\int_{a}^{b}\left|B_{2 r-1}^{*}\left(\frac{(a+b) / 2-t}{b-a}\right)\right|^{q} d t\right)^{1 / q}\left\|f^{(2 r-1)}\right\|_{p} \\
& \quad=\frac{(b-a)^{2 r-1+1 / q}}{(2 r-1) !}\left(\int_{0}^{1}\left|B_{2 r-1}(t)\right|^{q} d t\right)^{1 / q}\left\|f^{(2 r-1)}\right\|_{p} .
\end{aligned}
$$

Now apply the above estimate to the formula (2.6) to get (3.13). We get the inequalities (3.14) and (3.15) by a similar argument, using (2.7) and (2.8), respectively.

COROLlaRY 3.11. Let $f:[a, b] \rightarrow \mathbb{B}$ be such that $f^{(n)} \in L_{\infty}[a, b]$ for some $n \geq 1$ and assume the notation established by (2.1). If $n=2 r-1, r \geq 1$, then

$$
\left|\int_{a}^{b} f(t) d t-(b-a) f\left(\frac{a+b}{2}\right)+T_{r-1}(f)\right| \leq \frac{(b-a)^{2 r}}{(2 r) !} 4\left(1-2^{-2 r}\right)\left|B_{2 r}\right|\left\|f^{(2 r-1)}\right\|_{\infty} .
$$

If $n=2 r, r \geq 1$, then

$$
\left|\int_{a}^{b} f(t) d t-(b-a) f\left(\frac{a+b}{2}\right)+T_{r-1}(f)\right| \leq \frac{(b-a)^{2 r+1}}{(2 r) !}\left(1-2^{1-2 r}\right)\left|B_{2 r}\right|\left\|f^{(2 r)}\right\|_{\infty} .
$$

Also, we have

$$
\begin{aligned}
& \left|\int_{a}^{b} f(t) d t-(b-a) f\left(\frac{a+b}{2}\right)+T_{r}(f)\right| \\
& \quad \leq \frac{(b-a)^{2 r+1}}{(2 r) !} \int_{0}^{1}\left|B_{2 r}(t)\right| d t\left\|f^{(2 r)}\right\|_{\infty} \leq \frac{(b-a)^{2 r+1}}{(2 r) !} 2\left|B_{2 r}\right|\left\|f^{(2 r)}\right\|_{\infty} .
\end{aligned}
$$

PROOF. Apply Theorem 3.10 with $p=\infty$ and $q=1$, and use the same calculation for $\int_{0}^{1}\left|B_{2 r-1}(t)\right| d t, \int_{0}^{1}\left|B_{2 r}(t)-B_{2 r}(1 / 2)\right| d t$ and $\int_{0}^{1}\left|B_{2 r}(t)\right| d t$ as in the proof of Theorem 3.1.

COROLlaRY 3.12. Assume the pair $(p, q)$ is as in Theorem 3.10. Let $f:[a, b] \rightarrow$ $\mathbb{R}$ be a given function. If $f^{\prime} \in L_{p}[a, b]$, then

$$
\left|\int_{a}^{b} f(t) d t-(b-a) f\left(\frac{a+b}{2}\right)\right| \leq \frac{(b-a)^{1+1 / q}}{2(q+1)^{1 / q}}\left\|f^{\prime}\right\|_{p} .
$$

If $f^{\prime \prime} \in L_{p}[a, b]$, then

$$
\left|\int_{a}^{b} f(t) d t-(b-a) f\left(\frac{a+b}{2}\right)\right| \leq \frac{(b-a)^{2+1 / q}}{8(2 q+1)^{1 / q}}\left\|f^{\prime \prime}\right\|_{p} .
$$

https://doi.org/10.1017/S144618110000835X Published online by Cambridge University Press 
PROOF. To obtain the first inequality, apply (3.13) with $r=1$ and note that

$$
\int_{0}^{1}\left|B_{1}(t)\right|^{q} d t=\int_{0}^{1}|t-1 / 2|^{q} d t=\frac{1}{2^{q}(q+1)} .
$$

To obtain the second inequality, apply (3.14) with $r=1$ and note that

$$
\begin{aligned}
\int_{0}^{1}\left|B_{2}(t)-B_{2}(1 / 2)\right|^{q} d t & =\int_{0}^{1}\left|t^{2}-t+1 / 4\right|^{q} d t \\
& =2 \int_{1 / 2}^{1}(t-1 / 2)^{2 q} d t=\frac{1}{4^{q}(2 q+1)} .
\end{aligned}
$$

REMARK 5. For $p=\infty, q=1$, the inequalities established in Corollary 3.12 become

$$
\left|\int_{a}^{b} f(t) d t-(b-a) f\left(\frac{a+b}{2}\right)\right| \leq \frac{(b-a)^{2}}{4}\left\|f^{\prime}\right\|_{\infty}
$$

and

$$
\left|\int_{a}^{b} f(t) d t-(b-a) f\left(\frac{a+b}{2}\right)\right| \leq \frac{(b-a)^{3}}{24}\left\|f^{\prime \prime}\right\|_{\infty} .
$$

This second inequality coincides with the first inequality of (1.1). The second inequality established in Corollary 3.12 coincides with the second inequality in (1.1).

COROLlaRY 3.13. Let $f:[a, b] \rightarrow \mathbb{R}$ be a given function. If $f^{\prime \prime} \in L_{\infty}[a, b]$, then

$$
\left|\int_{a}^{b} f(t) d t-(b-a) f\left(\frac{a+b}{2}\right)-\frac{(b-a)^{2}}{24}\left[f^{\prime}(b)-f^{\prime}(a)\right]\right| \leq \frac{(b-a)^{3}}{18 \sqrt{3}}\left\|f^{\prime \prime}\right\|_{\infty} .
$$

If $f^{\prime \prime \prime} \in L_{\infty}[a, b]$, then

$$
\left|\int_{a}^{b} f(t) d t-(b-a) f\left(\frac{a+b}{2}\right)-\frac{(b-a)^{2}}{24}\left[f^{\prime}(b)-f^{\prime}(a)\right]\right| \leq \frac{(b-a)^{4}}{192}\left\|f^{\prime \prime \prime}\right\|_{\infty} .
$$

If $f^{\prime \prime \prime \prime} \in L_{\infty}[a, b]$, then

$$
\left|\int_{a}^{b} f(t) d t-(b-a) f\left(\frac{a+b}{2}\right)-\frac{(b-a)^{2}}{24}\left[f^{\prime}(b)-f^{\prime}(a)\right]\right| \leq \frac{7(b-a)^{5}}{5760}\left\|f^{\prime \prime \prime \prime}\right\|_{\infty} .
$$

PROOF. The result follows from Corollary 3.11 by an argument analogous to that used to obtain Corollary 3.3 from Theorem 3.1. 
COROLlaRY 3.14. Let $f:[a, b] \rightarrow \mathbb{R}$ be such that $f^{(n)} \in L_{2}[a, b]$ for some $n \geq 1$ and assume the notation established by (2.1). If $n=2 r-1, r \geq 1$, then

$$
\begin{aligned}
& \left|\int_{a}^{b} f(t) d t-(b-a) f\left(\frac{a+b}{2}\right)+T_{r-1}(f)\right| \\
& \quad \leq(b-a)^{2 r-1 / 2}\left(\frac{\left|B_{4 r-2}\right|}{(4 r-2) !}\right)^{1 / 2}\left\|f^{(2 r-1)}\right\|_{2} .
\end{aligned}
$$

If $n=2 r, r \geq 1$, then

$$
\begin{aligned}
& \left|\int_{a}^{b} f(t) d t-(b-a) f\left(\frac{a+b}{2}\right)+T_{r-1}(f)\right| \\
& \quad \leq(b-a)^{2 r+1 / 2}\left(\frac{\left|B_{4 r}\right|}{(4 r) !}+\frac{\left(1-2^{1-2 r}\right)^{2} B_{2 r}^{2}}{(2 r) !^{2}}\right)^{1 / 2}\left\|f^{(2 r)}\right\|_{2} .
\end{aligned}
$$

Also, we have

$$
\begin{gathered}
\left|\int_{a}^{b} f(t) d t-(b-a) f\left(\frac{a+b}{2}\right)+T_{r}(f)\right| \\
\quad \leq(b-a)^{2 r+1 / 2}\left(\frac{\left|B_{4 r}\right|}{(4 r) !}\right)^{1 / 2}\left\|f^{(2 r)}\right\|_{2} .
\end{gathered}
$$

PROOF. The Bernoulli polynomials satisfy the relations

and

$$
\int_{0}^{1} B_{n}(t) d t=0, \quad n \geq 1
$$

$$
\int_{0}^{1} B_{n}(t) B_{m}(t) d t=(-1)^{n-1} \frac{n ! m !}{(n+m) !} B_{n+m}, \quad n, m \geq 1
$$

(see $[1,23.1 .11,23.1 .12])$. Therefore we have

$$
\int_{0}^{1} B_{n}^{2}(t) d t=(-1)^{n-1} \frac{n !^{2}}{(2 n) !} B_{2 n}=\frac{n !^{2}}{(2 n) !}\left|B_{2 n}\right|
$$

and

$$
\begin{aligned}
& \int_{0}^{1}\left|B_{2 r}(t)-B_{2 r}(1 / 2)\right|^{2} d t \\
& \quad=\int_{0}^{1} B_{2 r}(t)^{2} d t-2 B_{2 r}(1 / 2) \int_{0}^{1} B_{2 r}(t) d t+B_{2 r}^{2}(1 / 2) \\
& \quad=\frac{(2 r) !^{2}}{(4 r) !}\left|B_{4 r}\right|+B_{2 r}^{2}(1 / 2)=\frac{(2 r) !^{2}}{(4 r) !}\left|B_{4 r}\right|+\left(1-2^{1-2 r}\right)^{2} B_{2 r}^{2} .
\end{aligned}
$$


Apply Theorem 3.10 with $p=q=2$. Using (3.19) with $n=2 r-1$ and (3.13), we get (3.16). Similarly, using (3.20) and (3.14), we get (3.17), while (3.18) follows from (3.15) and (3.19) with $n=2 r$.

COROLLARY 3.15. If $f^{\prime} \in L_{2}[a, b]$, then

$$
\left|\int_{a}^{b} f(t) d t-(b-a) f\left(\frac{a+b}{2}\right)\right| \leq \frac{(b-a)^{3 / 2}}{2 \sqrt{3}}\left\|f^{\prime}\right\|_{2}
$$

If $f^{\prime \prime} \in L_{2}[a, b]$, then

$$
\left|\int_{a}^{b} f(t) d t-(b-a) f\left(\frac{a+b}{2}\right)\right| \leq \frac{(b-a)^{5 / 2}}{8 \sqrt{5}}\left\|f^{\prime \prime}\right\|_{2} .
$$

PrOOF. Apply (3.16) with $r=1$ and (3.17) with $r=1$, respectively.

COROLlaRY 3.16. If $f^{\prime \prime} \in L_{2}[a, b]$, then

$$
\left|\int_{a}^{b} f(t) d t-(b-a) f\left(\frac{a+b}{2}\right)-\frac{(b-a)^{2}}{24}\left[f^{\prime}(b)-f^{\prime}(a)\right]\right| \leq \frac{(b-a)^{5 / 2}}{12 \sqrt{5}}\left\|f^{\prime \prime}\right\|_{2} .
$$

If $f^{\prime \prime \prime} \in L_{2}[a, b]$, then

$$
\left|\int_{a}^{b} f(t) d t-(b-a) f\left(\frac{a+b}{2}\right)-\frac{(b-a)^{2}}{24}\left[f^{\prime}(b)-f^{\prime}(a)\right]\right| \leq \frac{(b-a)^{7 / 2}}{12 \sqrt{210}}\left\|f^{\prime \prime \prime}\right\|_{2} .
$$

If $f^{\prime \prime \prime \prime} \in L_{2}[a, b]$, then

$$
\begin{aligned}
& \left|\int_{a}^{b} f(t) d t-(b-a) f\left(\frac{a+b}{2}\right)-\frac{(b-a)^{2}}{24}\left[f^{\prime}(b)-f^{\prime}(a)\right]\right| \\
& \quad \leq \frac{(b-a)^{9 / 2}}{5760} \sqrt{\frac{535}{7}}\left\|f^{\prime \prime \prime \prime}\right\|_{2} .
\end{aligned}
$$

PROOF. Apply (3.18) with $r=1$, (3.16) with $r=2$ and (3.17) with $r=2$, respectively.

\section{Error estimates for some quadrature formulae}

Let us divide the interval $[a, b]$ into $v$ subintervals of equal length $h=(b-a) / v$. Under appropriate assumptions on $f:[a, b] \rightarrow \mathbb{R}$, we consider the repeated Euler midpoint formula

$$
\int_{a}^{b} f(t) d t=S_{\nu}(f)-\tau_{r-1}(\nu, f)+\rho_{\nu}(f)
$$


where

$$
S_{\nu}(f):=h \sum_{i=1}^{\nu} f\left(a+(2 i-1) \frac{h}{2}\right)
$$

and

$$
\tau_{m}(\nu, f):=\sum_{k=1}^{m} \frac{h^{2 k}}{(2 k) !} B_{2 k}\left(\frac{1}{2}\right)\left[f^{(2 k-1)}(b)-f^{(2 k-1)}(a)\right]
$$

with the convention that $\tau_{0}(\nu, f)=0$.

Since

$$
\sum_{i=1}^{\nu}\left[f^{(2 k-1)}(a+i h)-f^{(2 k-1)}(a+(i-1) h)\right]=f^{(2 k-1)}(b)-f^{(2 k-1)}(a),
$$

the remainder $\rho_{\nu}(f)$ can be written in the form

$$
\rho_{\nu}(f)=\sum_{i=1}^{\nu} \rho_{\nu}(f ; i)
$$

where, for $i=1, \ldots, v$,

$$
\begin{aligned}
\rho_{\nu}(f ; i)= & \int_{a+(t-1) h}^{a+i h} f(t) d t-h f\left(a+(2 i-1) \frac{h}{2}\right) \\
& +\sum_{k=1}^{r-1} \frac{h^{2 k}}{(2 k) !} B_{2 k}\left(\frac{1}{2}\right)\left[f^{(2 k-1)}(a+i h)-f^{(2 k-1)}(a+(i-1) h)\right] .
\end{aligned}
$$

We shall apply the results from the preceding section to obtain some estimates for the remainder $\rho_{\nu}(f)$.

THEOREM 4.1. Let $f:[a, b] \rightarrow \mathbb{R}$ be such that $f^{(n-1)}$ is an L-Lipschitzian function on $[a, b]$ for some $n \geq 1$. Then for $n=2 r-1, r \geq 1$, we have

$$
\left|\rho_{\nu}(f)\right| \leq \frac{v h^{2 r}}{(2 r) !} 4\left(1-2^{-2 r}\right)\left|B_{2 r}\right| L
$$

while for $n=2 r, r \geq 1$, we have

$$
\left|\rho_{\nu}(f)\right| \leq \frac{v h^{2 r+1}}{(2 r) !}\left(1-2^{1-2 r}\right)\left|B_{2 r}\right| L .
$$

Proof. Using (3.1) we get for $i=1, \ldots, v$ that

$$
\left|\rho_{\nu}(f ; i)\right| \leq \frac{h^{2 r}}{(2 r) !} 4\left(1-2^{-2 r}\right)\left|B_{2 r}\right| L .
$$


By the triangle inequality, we get from (4.4) that

$$
\begin{aligned}
\left|\rho_{\nu}(f)\right| \leq \sum_{i=1}^{\nu}\left|\rho_{\nu}(f ; i)\right| & \leq \sum_{i=1}^{v} \frac{h^{2 r}}{(2 r) !} 4\left(1-2^{-2 r}\right)\left|B_{2 r}\right| L \\
& =\frac{v h^{2 r}}{(2 r) !} 4\left(1-2^{-2 r}\right)\left|B_{2 r}\right| L
\end{aligned}
$$

which proves the first assertion. Further, using (3.2), we get the estimate

$$
\left|\rho_{\nu}(f ; i)\right| \leq \frac{h^{2 r+1}}{(2 r) !}\left(1-2^{1-2 r}\right)\left|B_{2 r}\right| L
$$

which holds for $i=1, \ldots, v$. Using (4.4) and the triangle inequality, we prove the second assertion in the same way as we did the first one.

THEOREM 4.2. Let $f:[a, b] \rightarrow \mathbb{B}$ be such that $f^{(n-1)}$ is a continuous function of bounded variation on $[a, b]$ for some $n \geq 1$. Then for $n=2 r-1, r \geq 2$, we have

$$
\begin{aligned}
\left|\rho_{\nu}(f)\right| & \leq \frac{h^{2 r-1}}{(2 r-1) !} \max _{t \in[0,1]}\left|B_{2 r-1}(t)\right| V_{a}^{b}\left(f^{(2 r-2)}\right) \\
& \leq \frac{2 h^{2 r-1}}{(2 \pi)^{2 r-1}\left(1-2^{2-2 r}\right)} V_{a}^{b}\left(f^{(2 r-2)}\right)
\end{aligned}
$$

and the first inequality holds for $r=1$ too. Also, for $n=2 r, r \geq 1$, we have

$$
\left|\rho_{v}(f)\right| \leq \frac{2 h^{2 r}}{(2 r) !}\left(1-2^{-2 r}\right)\left|B_{2 r}\right| V_{a}^{b}\left(f^{(2 r-1)}\right) .
$$

ProOF. Applying (3.9), we get for $i=1, \ldots, v$ that

$$
\begin{aligned}
\left|\rho_{\nu}(f ; i)\right| & \leq \frac{h^{2 r-1}}{(2 r-1) !} \max _{t \in[0,1]}\left|B_{2 r-1}(t)\right| V_{a+(i-1) h}^{a+i h}\left(f^{(2 r-2)}\right) \\
& \leq \frac{2 h^{2 r-1}}{(2 \pi)^{2 r-1}\left(1-2^{2-2 r}\right)} V_{a+(i-1) h}^{a+i h}\left(f^{(2 r-2)}\right) .
\end{aligned}
$$

From (4.4), we have by the triangle inequality that

$$
\begin{aligned}
\left|\rho_{\nu}(f)\right| \leq \sum_{i=1}^{\nu}\left|\rho_{\nu}(f ; i)\right| & \leq \frac{h^{2 r-1}}{(2 r-1) !} \max _{r \in[0,1]}\left|B_{2 r-1}(t)\right| \sum_{i=1}^{\nu} V_{a+(i-1) h}^{a+i h}\left(f^{(2 r-2)}\right) \\
& =\frac{h^{2 r-1}}{(2 r-1) !} \max _{i \in[0,1]}\left|B_{2 r-1}(t)\right| V_{a}^{b}\left(f^{(2 r-2)}\right) \\
& \leq \frac{2 h^{2 r-1}}{(2 \pi)^{2 r-1}\left(1-2^{2-2 r}\right)} V_{a}^{b}\left(f^{(2 r-2)}\right),
\end{aligned}
$$


which proves the first assertion. To prove the second, we use (3.10) to obtain the estimate

$$
\left|\rho_{\nu}(f ; i)\right| \leq \frac{2 h^{2 r}}{(2 r) !}\left(1-2^{-2 r}\right)\left|B_{2 r}\right| V_{a+(i-1) h}^{a+i h}\left(f^{(2 r-1)}\right)
$$

for $i=1, \ldots, v$. From (4.4) we get

$$
\begin{aligned}
\left|\rho_{\nu}(f)\right| \leq \sum_{i=1}^{\nu}\left|\rho_{\nu}(f ; i)\right| & \leq \frac{2 h^{2 r}}{(2 r) !}\left(1-2^{-2 r}\right)\left|B_{2 r}\right| \sum_{i=1}^{\nu} V_{a+(i-1) h}^{a+i h}\left(f^{(2 r-1)}\right) \\
& =\frac{2 h^{2 r}}{(2 r) !}\left(1-2^{-2 r}\right)\left|B_{2 r}\right| V_{a}^{b}\left(f^{(2 r-1)}\right)
\end{aligned}
$$

which proves the second assertion.

COROLlARY 4.3. Let $f:[a, b] \rightarrow \mathbb{R}$ be such that $f^{(n)} \in L_{1}[a, b]$, for some $n \geq 1$. Then for $n=2 r-1, r \geq 2$, we have

$$
\begin{aligned}
\left|\rho_{\nu}(f)\right| & \leq \frac{h^{2 r-1}}{(2 r-1) !} \max _{t \in[0.1]}\left|B_{2 r-1}(t)\right|\left\|f^{(2 r-1)}\right\|_{1} \\
& \leq \frac{2 h^{2 r-1}}{(2 \pi)^{2 r-1}\left(1-2^{2-2 r}\right)}\left\|f^{(2 r-1)}\right\|_{1}
\end{aligned}
$$

and the first inequality holds for $r=1$ too. Also, for $n=2 r, r \geq 1$, we have

$$
\left|\rho_{\nu}(f)\right| \leq \frac{2 h^{2 r}}{(2 r) !}\left(1-2^{-2 r}\right)\left|B_{2 r}\right|\left\|f^{(2 r)}\right\|_{1} .
$$

PROOF. Note that $f^{(n-1)}$ has bounded variation on $[a, b]$,

$$
V_{a}^{b}\left(f^{(n-1)}\right)=\int_{a}^{b}\left|f^{(n)}(t)\right| d t=\left\|f^{(n)}\right\|_{t},
$$

and then apply Theorem 4.2 to obtain the inequalities stated in the corollary.

THEOREM 4.4. Let $(p, q)$ be a pair of conjugate exponents, that is, $1<p, q<\infty$, $1 / p+1 / q=1$ or $p=\infty, q=1$. Let $f:[a, b] \rightarrow \mathbb{R}$ be such that $f^{(n)} \in L_{p}[a, b]$ for some $n \geq 1$. Then for $n=2 r-1, r \geq 1$, we have

$$
\left|\rho_{\nu}(f)\right| \leq \frac{\nu h^{2 r-1+1 / q}}{(2 r-1) !}\left(\int_{0}^{1}\left|B_{2 r-1}(t)\right|^{q} d t\right)^{1 / q}\left\|f^{(2 r-1)}\right\|_{p},
$$

while for $n=2 r, r \geq 1$, we have

$$
\left|\rho_{\nu}(f)\right| \leq \frac{v h^{2 r+1 / q}}{(2 r) !}\left(\int_{0}^{1}\left|B_{2 r}(t)-B_{2 r}\left(\frac{1}{2}\right)\right|^{q} d t\right)^{1 / q}\left\|f^{(2 r)}\right\|_{p} .
$$


ProOF. For $i=1, \ldots, v$ consider the function

$$
g_{i}(t)=f^{(n)}(t), \quad t \in[a+(i-1) h, a+i h] .
$$

Obviously we have $\left\|g_{i}\right\|_{p} \leq\left\|f^{(n)}\right\|_{p}$, where the norm $\left\|g_{i}\right\|_{p}$ is taken over the interval $[a+(i-1) h, a+i h]$, while the norm $\left\|f^{(n)}\right\|_{p}$ is taken over the interval $[a, b]$. Using the above inequality, we get for $i=1, \ldots, \nu$ that

$$
\begin{aligned}
\left|\rho_{\nu}(f ; i)\right| & \leq \frac{h^{2 r-1+1 / q}}{(2 r-1) !}\left(\int_{0}^{1}\left|B_{2 r-1}(t)\right|^{q} d t\right)^{1 / q}\left\|g_{1}\right\|_{p} \\
& \leq \frac{h^{2 r-1+1 / q}}{(2 r-1) !}\left(\int_{0}^{1}\left|B_{2 r-1}(t)\right|^{q} d t\right)^{1 / q}\left\|f^{(2 r-1)}\right\|_{p}
\end{aligned}
$$

using (3.13) in the case $n=2 r-1$, and

$$
\begin{aligned}
\left|\rho_{\nu}(f ; i)\right| & \leq \frac{h^{2 r+1 / q}}{(2 r) !}\left(\int_{0}^{1}\left|B_{2 r}(t)-B_{2 r}(1 / 2)\right|^{q} d t\right)^{1 / q}\left\|g_{1}\right\|_{p} \\
& \leq \frac{h^{2 r+1 / q}}{(2 r) !}\left(\int_{0}^{1}\left|B_{2 r}(t)-B_{2 r}(1 / 2)\right|^{q} d t\right)^{1 / q}\left\|f^{(2 r)}\right\|_{p},
\end{aligned}
$$

using (3.14) in the case $n=2 r$. The rest of the argument is the same as for the preceding theorems.

COROLlaRY 4.5. Let $f:[a, b] \rightarrow \mathbb{R}$ be such that $f^{(n)} \in L_{\infty}[a, b]$ for some $n \geq 1$. Then for $n=2 r-1, r \geq 1$, we have

while for $n=2 r, r \geq 1$, we have

$$
\left|\rho_{\nu}(f)\right| \leq \frac{\nu h^{2 r}}{(2 r) !} 4\left(1-2^{-2 r}\right)\left|B_{2 r}\right|\left\|f^{(2 r-1)}\right\|_{\infty},
$$

$$
\left|\rho_{\nu}(f)\right| \leq \frac{\nu h^{2 r+1}}{(2 r) !}\left(1-2^{1-2 r}\right)\left|B_{2 r}\right|\left\|f^{(2 r)}\right\|_{\infty} .
$$

Proof. Apply Theorem 4.4 with $p=\infty$.

COROLlaRY 4.6. Let $f:[a, b] \rightarrow \mathbb{R}$ be such that $f^{(n)} \in L_{2}[a, b]$ for some $n \geq 1$. Then for $n=2 r-1, r \geq 1$, we have

$$
\left|\rho_{\nu}(f)\right| \leq \nu h^{2 r-1 / 2}\left(\frac{\left|B_{4 r-2}\right|}{(4 r-2) !}\right)^{1 / 2}\left\|f^{(2 r-1)}\right\|_{2},
$$

while for $n=2 r-1, r \geq 1$, we have

$$
\left|\rho_{v}(f)\right| \leq v h^{2 r+1 / 2}\left(\frac{\left|B_{4 r}\right|}{(4 r) !}+\frac{\left(1-2^{1-2 r}\right)^{2} B_{2 r}^{2}}{(2 r) !^{2}}\right)^{1 / 2}\left\|f^{(2 r)}\right\|_{2}
$$


PROOF. Apply Theorem 4.4 with $p=2$.

Instead of the repeated Euler midpoint formula (4.1), under appropriate assumptions on $f:[a, b] \rightarrow \mathbb{R}$, we can consider the repeated modified Euler midpoint formula

$$
\int_{a}^{b} f(t) d t=S_{\nu}(f)-\tau_{r}(f)+\sigma_{v}(f),
$$

where $\nu$ and $h=(b-a) / \nu$ are as before, $S_{\nu}(f)$ is given by (4.2), and $\tau_{r}(f)$ is defined by (4.3). In this case, the remainder $\sigma_{v}(f)$ can be written in the form

$$
\sigma_{\nu}(f)=\sum_{i=1}^{\nu} \sigma_{\nu}(f ; i)
$$

where, for $i=1, \ldots, \nu$,

$$
\begin{aligned}
\sigma_{\nu}(f ; i) & =\int_{a+(t-1) h}^{a+i h} f(t) d t-h f\left(a+(2 i-1) \frac{h}{2}\right) \\
& +\sum_{k=1}^{r} \frac{h^{2 k}}{(2 k) !} B_{2 k}\left(\frac{1}{2}\right)\left[f^{(2 k-1)}(a+i h)-f^{(2 k-1)}(a+(i-1) h)\right] .
\end{aligned}
$$

Here we give the estimates for the remainder $\sigma_{\nu}(f)$, using the same argument as that used for $\rho_{v}(f)$. We omit the details.

THEOREM 4.7. Let $f:[a, b] \rightarrow \mathbb{R}$ be such that $f^{(2 r-1)}$ is an L-Lipschitzian function on $[a, b]$, for some $r \geq 1$. Then

$$
\left|\sigma_{v}(f)\right| \leq \frac{v h^{2 r+1}}{(2 r) !} \int_{0}^{1}\left|B_{2 r}(t)\right| d t L \leq \frac{v h^{2 r+1}}{(2 r) !} 2\left|B_{2 r}\right| L .
$$

PROOF. As in Theorem 4.1, using (3.3) and (4.5).

THEOREM 4.8. Let $f:[a, b] \rightarrow \mathbb{R}$ be such that $f^{(2 r-1)}$ is a continuous function of bounded variation on $[a, b]$, for some $r \geq 1$. Then

$$
\left|\sigma_{\nu}(f)\right| \leq \frac{h^{2 r}}{(2 r) !}\left|B_{2 r}\right| V_{a}^{b}\left(f^{(2 r-1)}\right)
$$

PROOF. As in Theorem 4.2, using (3.11) and (4.5).

COROLlaRY 4.9. Let $f:[a, b] \rightarrow \mathbb{R}$ be such that $f^{(2 r)} \in L_{1}[a, b]$, for some $r \geq 1$. Then $\left|\sigma_{v}(f)\right| \leq\left(h^{2 r} /(2 r) !\right)\left|B_{2 r}\right|\left\|f^{(2 r)}\right\|_{1}$. 
PROOF. Note that $f^{(2 r-1)}$ has bounded variation on $[a, b]$,

$$
V_{a}^{b}\left(f^{(2 r-1)}\right)=\int_{a}^{b}\left|f^{(2 r)}(t)\right| d t=\left\|f^{(2 r)}\right\|_{1}
$$

and then apply Theorem 4.8 .

THEOREM 4.10. Lef $(p, q)$ be a pair of conjugate exponents, that is, $1<p, q<\infty$, $1 / p+1 / q=1$ or $p=\infty, q=1$. Let $f:[a, b] \rightarrow \mathbb{R}$ be such that $f^{(2 r)} \in L_{p}[a, b]$, for some $r \geq 1$. Then

$$
\left|\sigma_{\nu}(f)\right| \leq \frac{\nu h^{2 r+1 / q}}{(2 r) !}\left(\int_{0}^{1}\left|B_{2 r}(t)\right|^{q} d t\right)^{1 / q}\left\|f^{(2 r)}\right\|_{p} .
$$

PROOF. As in Theorem 4.4, using (3.15) and (4.5).

COROLLARY 4.11. Let $f:[a, b] \rightarrow \mathbb{R}$ be such that $f^{(2 r)} \in L_{\infty}[a, b]$, for some $r \geq 1$. Then

$$
\left|\sigma_{v}(f)\right| \leq \frac{\nu h^{2 r+1}}{(2 r) !} \int_{0}^{1}\left|B_{2 r}(t)\right| d t\left\|f^{(2 r)}\right\|_{\infty} \leq \frac{\nu h^{2 r+1}}{(2 r) !} 2\left|B_{2 r}\right|\left\|f^{(2 r)}\right\|_{\infty} .
$$

Proof. Apply Theorem 4.10 with $p=\infty$ and use (3.8).

COROLLARY 4.12. Let $f:[a, b] \rightarrow \mathbb{R}$ be such that $f^{(2 r)} \in L_{2}[a, b]$, for some $r \geq 1$. Then $\left|\sigma_{\nu}(f)\right| \leq \nu h^{2 r+1 / 2}\left(\left|B_{4 r}\right| /(4 r) !\right)^{1 / 2}\left\|f^{(2 r)}\right\|_{2}$.

Proof. Apply Theorem 4.10 with $p=2$.

\section{References}

[1] M. Abramowitz and I. A. Stegun (eds.), Handbook of mathematical functions with formulae, graphs and mathematical tables, Applied Math. Series 55, 4th printing (National Bureau of Standards, Washington, 1965).

[2] I. S. Berezin and N. P. Zhidkov, Computing methods Vol. I (Pergamon Press, Oxford, 1965).

[3] P. C. Cerone and S. S Dragomir, "Midpoint type rules from an inequalities point of view", RGMIA Research Report Collection 2(7), Article 1, 1999.

[4] Lj. Dedić, M. Matić and J. Pečarić, "On generalizations of Ostrowski inequality via some Euler-type identities", Math. Inequal. Appl. 3 (2000) 337-353.

[5] S. S. Dragomir, "On the midpoint quadrature formula for Lipschitzian mappings and applications", Kragujevac J. Math. 22 (2000) 5-11.

[6] S. S. Dragomir, P. Cerone and A. Sofo, "Some remarks on the midpoint rule in numerical integration", RGMIA, Research Report Collection I(2), Article 4, 1998.

[7] V. I. Krylov, Approximate calculation of integrals (Macmillan, New York, 1962). 